12,17

\title{
Термический отжиг дефектов Стоуна-Уэльса в фуллеренах и нанотрубках
}

\author{
(c) А.И. Подливаев ${ }^{1,2}$, Л.А. Опенов ${ }^{1}$ \\ ${ }^{1}$ Национальный исследовательский ядерный университет „МИФИ“, \\ Москва, Россия \\ ${ }^{2}$ Научно-исследовательский институт проблем развития научно-образовательного потенциала молодежи, \\ Москва, Россия \\ E-mail: AIPodlivayev@mephi.ru
}

(Поступила в Редакцию 11 апреля 2017 г.)

\begin{abstract}
Методом молекулярной динамики изучен термоактивированный отжиг топологических дефектов Стоуна-Уэльса в углеродных фуллеренах и нанотрубках. Показано, что температурные зависимости характерных времен отжига подчиняются закону Аррениуса. Определены значения соответствующих энергий активации и частотных факторов. Полученные результаты сопоставлены с данными моделирования поверхностей потенциальной энергии.
\end{abstract}

Работа выполнена при поддержке Министерства образования и науки РФ в рамках Программы повышения конкурентоспособности НИЯУ МИФИ, договор № 02.А03.21.0005. Работа поддержана грантом РФФИ № 15-02-02764.

DOI: 10.21883/FTT.2018.01.45303.124

\section{1. Введение}

В низкоразмерных углеродных структурах (фуллеренах [1], нанотрубках [2], графене [3] и др. [4]) одним из основных типов дефектов являются топологические дефекты Стоуна-Уэльса (Stone-Wales, SW), которые образуются при трансформациях SW - поворотах связей $\mathrm{C}-\mathrm{C}$ на угол $\approx 90^{\circ}[5]$. Дефекты SW могут играть как отрицательную, так и конструктивную роль. Например, накопление этих дефектов в фуллерене $\mathrm{C}_{60}$ при сильном нагреве приводит в конечном итоге к фрагментации кластера [6], а их присутствие в углеродных нанотрубках (УНТ) снижает механическую жесткость последних $[7,8]$. С другой стороны, будучи центрами предпочтительной адсорбции различных химических групп и элементов, дефекты SW могут существенно улучшить функциональные характеристики УНТ и фуллеренов $[9,10]$. Таким образом, в зависимости от целей конкретного эксперимента и/или предполагаемого направления практического использования той или иной углеродной наноструктуры может оказаться желательным как уменьшение, так и увеличение количества дефектов SW в ней.

Для создания дефектов SW требуются сильные механические деформации (например, излом УНТ [11]) или облучение частицами с высокой энергией. Что касается очистки наноструктуры от этих дефектов, то наиболее практичным представляется ее нагрев до температур, при которых тепловая энергия достаточно велика для отжига имеющихся в образце дефектов SW, но недостаточна для термоактивированного образования новых дефектов (а тем более - для разрушения наноструктуры). Целью настоящей работы является компьютерное моделирование процесса отжига дефектов SW и опреде- ление температурных зависимостей характерных времен отжига. В качестве объектов исследования мы выбрали фуллерен $\mathrm{C}_{60}$ и одностенную УНТ $(10,0)$.

\section{2. Методы расчета}

Межатомные взаимодействия описывались в рамках неортогональной модели сильной связи [12], которая уступает по точности первопринципным методам, но при этом гораздо менее требовательна к вычислительным ресурсам, что позволяет проследить эволюцию системы из $\sim 100$ атомов в течение достаточно длительного (по атомным меркам) времени. Эта модель продемонстрировала свою применимость к численному исследованию самых различных углеродных наноструктур (см. работы [13-16] и ссылки в них).

Для фуллерена $\mathrm{C}_{60}$ выбирались свободные граничные условия по всем направлениям. Для УНТ $(10,0)$ использовалась сверхъячейка с периодическими граничными условиями вдоль оси УНТ и свободными в двух поперечных направлениях. Период сверхъячейки определялся из условия минимума энергии равновесной конфигурации.

Моделирование временно́й эволюции фуллерена и УНТ проводилось методом молекулярной динамики (МД). Для численного интегрирования уравнений движения использовался алгоритм Верле с шагом по времени $0.3 \mathrm{fs}$ (детали см. в работе [17]). В исходных конфигурациях фуллерена и УНТ присутствовал один дефект SW. Время отжига этого дефекта мы принимали равным времени формирования бездефектной конфигурации за счет обратной трансформации SW. Для визуализации деталей процесса отжига мы запоминали координаты всех 
атомов каждые 10-100 шагов МД, а затем представляли полученные результаты в виде компьютерной анимации.

\section{3. Результаты и их обсуждение}

3.1. Фуллерен $\mathrm{C}_{60}$. В фуллерене $\mathrm{C}_{60}$ (рис. $\left.1, a\right)$ связи $\mathrm{C}-\mathrm{C}$ формируют 20 шестиугольников и 12 изолированных друг от друга пятиугольников. Дефект SW с наименьшей энергией образуется при повороте одной из общих для двух шестиугольников связей $\mathrm{C}-\mathrm{C}$. Дефектный изомер (рис. $1, b)$ содержит две пары смежных пятиугольников. Определенная нами энергия формирования этого изомера составила $E_{f}=1.34 \mathrm{eV}$, что близко к значениям $E_{f}=1.4-1.7 \mathrm{eV}$, полученным в рамках теории функционала плотности [18-23].

На рис. 2 изображена зависимость энергии $E$ кластера $\mathrm{C}_{60}$ от угла $\phi$ поворота связи $\mathrm{C}-\mathrm{C}$ при трансформации $\mathrm{SW}$. Максимум $E$ отвечает седловой точке поверхности потенциальной энергии (PES) кластера как функции координат составляющих его атомов. Он определяет высоты энергетических барьеров для формирования и отжига дефекта $\mathrm{SW}, U_{f}=5.24 \mathrm{eV}$ и $U_{a}=3.90 \mathrm{eV}$ соответственно.

Понижение температуры ведет к резкому увеличению времени протекания термоактивированных процессов (в том числе отжига) и соответственно требующегося для расчетов компьютерного времени. Поэтому мы выполняли моделирование отжига дефекта SW при высоких температурах $T=2900-4200 \mathrm{~K}$, а затем экстраполировали результаты на область более низких температур. Полученные данные представлены на рис. $3, a$. Видно, что в широком (около трех порядков величины) диапазоне значений времени отжига $\tau$ зависимость логарифма $\tau$ от обратной температуры достаточно хорошо аппроксимируется прямой линией. Это свидетельствует (вполне ожидаемо) о том, что отжиг дефекта SW описывается законом Аррениуса

$$
\frac{1}{\tau(T)}=A \exp \left[-\frac{E_{a}}{k_{\mathrm{B}} T}\right]
$$

где $E_{a}-$ энергия активации, которая обычно близка к высоте соответствующего энергетического барьера,

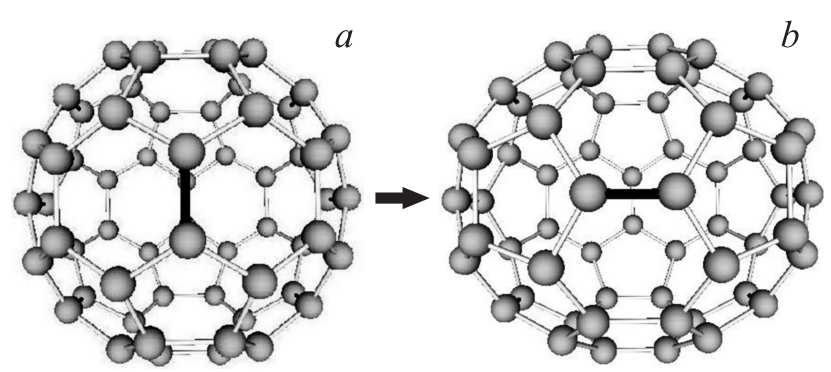

Рис. 1. Образование дефекта $\mathrm{SW}$ в фуллерене $\mathrm{C}_{60}$. Поворачивающаяся при трансформации SW связь C-C выделена черным цветом.

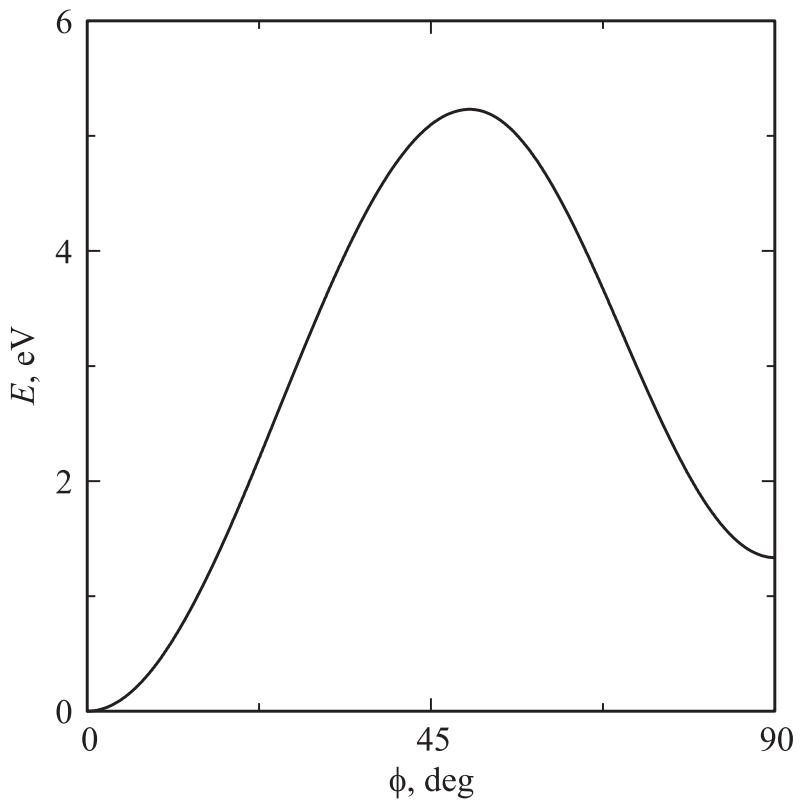

Рис. 2. Зависимость энергии $E$ кластера $\mathrm{C}_{60}$ от угла $\phi$ поворота связи С-C при трансформации SW (рис. 1). За начало отсчета принята энергия бездефектного фуллерена $E(\phi=0)$. Энергия формирования дефекта $E_{f}=E\left(\phi=90^{\circ}\right)-E(\phi=0)=1.34 \mathrm{eV}$. Барьер для формирования дефекта $U_{f}=E\left(\phi=50^{\circ}\right)-E(\phi=0)=5.24 \mathrm{eV}$. Барьер для отжига дефекта $U_{a}=E\left(\phi=50^{\circ}\right)-E\left(\phi=90^{\circ}\right)=3.90 \mathrm{eV}$.

$A-$ не зависящий (или слабо зависящий) от температуры частотный фактор с размерностью $\mathrm{s}^{-1}, k_{\mathrm{B}}-$ постоянная Больцмана.

Статистическая обработка данных „компьютерного эксперимента“ дает $E_{a}=4.27 \pm 0.54 \mathrm{eV}, \quad A=$ $=(2.66 \pm 0.45) \cdot 10^{16} \mathrm{~s}^{-1}$. Заметим, что среднее значение $E_{a}$ превышает высоту препятствующего отжигу барьера $U_{a}$ (см. рис. 2), а частотный фактор в $\sim 5$ раз больше величины $4.7 \cdot 10^{15} \mathrm{~s}^{-1}$, найденной нами по формуле Виньярда [24] на основании расчета спектров собственных колебаний кластера в стационарных точках PES. Причина такого несоответствия данных молекулярной динамики с результатами анализа PES заключается, по-видимому, в существовании нескольких (помимо рассмотренного нами) путей отжига дефекта $\mathrm{SW}$ с разными наборами $\left(U_{a}, A\right)$, что неудивительно, если принять во внимание наличие по меньшей мере двух различных путей формирования этого дефекта (см. работу [25] и ссылки в ней).

Как бы то ни было, определив значения параметров $E_{a}$ и $A$ в формуле (1), мы можем найти время отжига дефекта SW при температурах ниже рассмотренного диапазона. Например, $\tau \sim 10 \mathrm{~ms}$ при $T=1500 \mathrm{~K}$. Понижение температуры ведет к увеличению $\tau$ до $\sim 1 \mathrm{~s}$ при $T=1300 \mathrm{~K}, \sim 20 \min$ при $T=1100 \mathrm{~K}$ и т.д.

Отметим, что вместо ожидаемого отжига дефекта SW мы неоднократно наблюдали образование второго такого же дефекта. Это вязано с большим (около 30) 

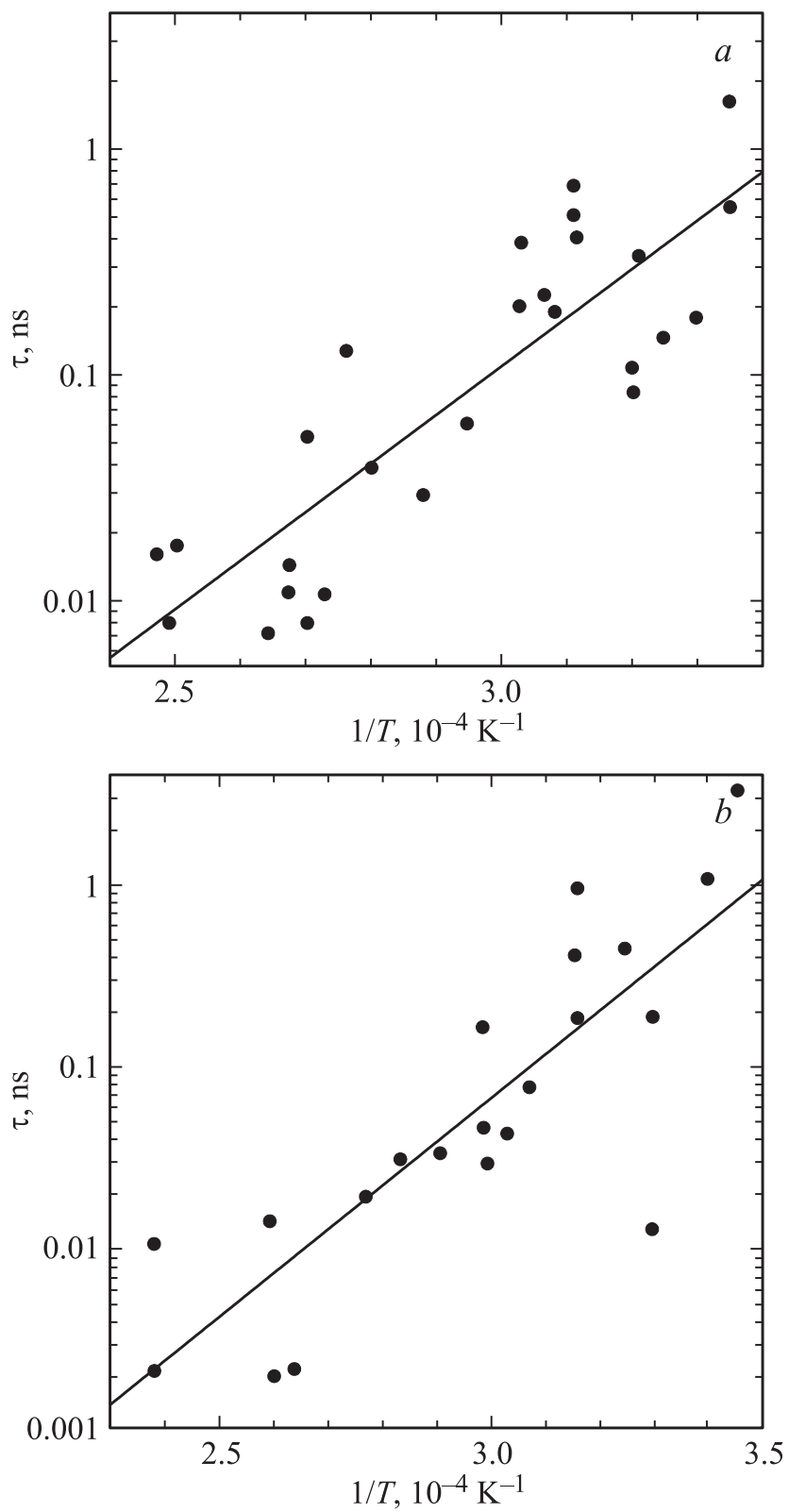

Рис. 3. Зависимости $(a)$ времени $\tau$ отжига дефекта SW в фуллерене $\mathrm{C}_{60}$ с одним таким дефектом и $(b)$ времени $\tau_{2}$ образования второго дефекта SW от обратной температуры $T$. Точки - результаты численного счета. Сплошные линии линейные аппроксимации методом наименьших квадратов.

числом путей формирования нового дефекта (то есть количеством общих для двух шестиугольников связей $\mathrm{C}-\mathrm{C})$. В результате, несмотря на более высокий барьер (см. рис. 2), вероятность $P_{+}$появления еще одного дефекта SW может оказаться сравнимой с вероятностью $P$-отжига одного единственного уже имеющегося в кластере дефекта или даже превысить ее. Температурная зависимость времени $\tau_{2}$ образования второго дефекта SW в фуллерене $\mathrm{C}_{60}$ представлена на рис. $3, b$. Она описывается формулой (1) с параметрами $E_{a}=4.78 \pm 0.77 \mathrm{eV}$, $A=(2.56 \pm 0.68) \cdot 10^{17} \mathrm{~s}^{-1}$. Используя значения энер- гий активации и частотных факторов, найденные для отжига и формирования дефекта SW, можно оценить вероятности $P_{-}$и $P_{+}$при различных температурах. Оказывается, что $P_{-} \sim P_{+}$при $T=2600 \mathrm{~K}$. При понижении температуры величина отношения $P_{-} / P_{+}$быстро увеличивается, достигая $\sim 10$ при $T=1300 \mathrm{~K}$ и $\sim 100$ при $T=850 \mathrm{~K}$. Следует понимать, что увеличение относительной вероятности отжига дефекта достигается при этом ценой увеличения времени его отжига.

3.2. Нанотрубка $(10,0)$. В УНТ $(10,0)$ имеется два типа неэквивалентных связей $\mathrm{C}-\mathrm{C}$, поэтому в ней возможно формирование двух типов различных дефектов SW: одни образуются при повороте параллельных
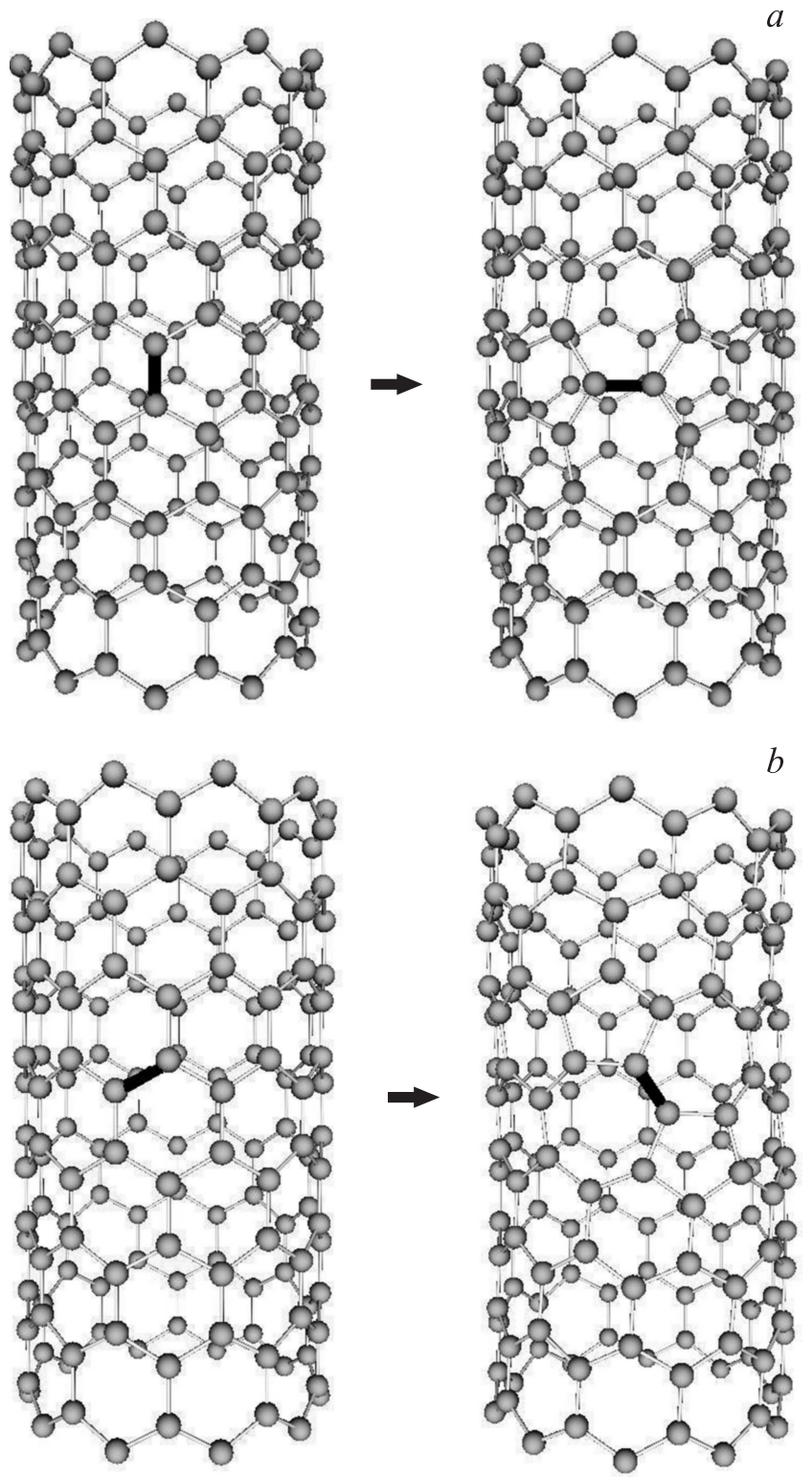

Рис. 4. Образование дефектов SW в УНТ $(10,0)$. При повороте параллельной оси УНТ связи С-C $(a)$ образуется дефект SWA, а при повороте направленной под углом к этой оси связи C-C $(b)$ - дефект SWB. Поворачивающиеся при трансформациях SW связи C-C выделены черным цветом. 


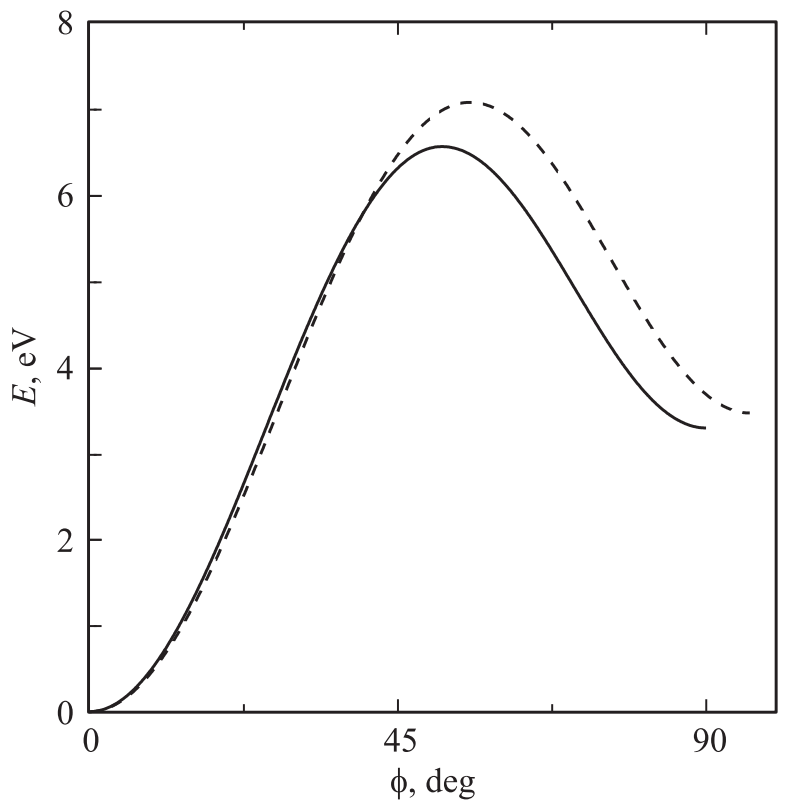

Рис. 5. То же, что на рис. 2, для дефектов SWA (сплошная линия) и SWB (штриховая линия) в УНТ $(10,0)$, см. рис. 4. Энергии формирования дефектов $E_{f}=E\left(\phi=90^{\circ}\right)$ - $E(\phi=0)=3.30 \mathrm{eV}$ для дефекта SWA и $E_{f}=E\left(\phi=96^{\circ}\right)$ $-E(\phi=0)=3.47 \mathrm{eV}$ для дефекта SWB. Барьеры для формирования дефектов $U_{f}=E\left(\phi=51^{\circ}\right)-E(\phi=0)=6.57 \mathrm{eV}$ для дефекта SWA и $U_{f}=E\left(\phi=56^{\circ}\right)-E(\phi=0)=7.08 \mathrm{eV}$ для дефекта SWB. Барьеры для отжига дефектов $U_{a}=E\left(\phi=51^{\circ}\right)-E\left(\phi=90^{\circ}\right)=3.27 \mathrm{eV}$ для дефекта SWA и $U_{a}=E\left(\phi=56^{\circ}\right)-E\left(\phi=96^{\circ}\right)=3.61 \mathrm{eV}$ для дефекта SWB.

оси УНТ связей $\mathrm{C}-\mathrm{C}$, см. рис. 4, $a$ (будем называть их дефектами SWA), а другие - при повороте связей $\mathrm{C}-\mathrm{C}$, направленных под углом к этой оси, см. рис. $4, b$ (дефекты SWB). Для энергии формирования дефектов SWA мы получили $E_{f}=3.30 \mathrm{eV}$, что согласуется с имеющимися в литературе значениями $E_{f}=3.25 \mathrm{eV}$ [26] и $2.88 \mathrm{eV}$ [27], а также с величиной $E_{f}=3.54 \mathrm{eV}$, рассчитанной по приведенной в работе [28] формуле, для $E_{f}$ в УНТ различного диаметра и хиральности. Для дефекта SWB мы нашли $E_{f}=3.47 \mathrm{eV}$, что на $\sim 1.5 \mathrm{eV}$ меньше, чем дает расчет по упомянутой формуле. Причина такого расхождения нам не вполне ясна, особенно если учесть неплохое соответствие для дефекта SWA. Высоты энергетических барьеров для формирования и отжига этих дефектов равны соответственно $U_{f}=6.57 \mathrm{eV}, U_{a}=3.27 \mathrm{eV}$ для дефекта SWA и $U_{f}=7.08 \mathrm{eV}, U_{a}=3.61 \mathrm{eV}$ для дефекта SWB (см. рис. 5).

Моделирование отжига проводилось при $T=$ $=3000-4900 \mathrm{~K}$. Полученные результаты представлены на рис. 6. Как и в случае фуллерена $\mathrm{C}_{60}$, температурные зависимости времен отжига обоих дефектов подчиняются закону Аррениуса (1). Статистический анализ дает следующие значения энергий активации отжига и частотных факторов: $E_{a}=3.00 \pm 0.28 \mathrm{eV}$, $A=(6.81 \pm 0.83) \cdot 10^{14} \mathrm{~s}^{-1}$ для дефекта SWA и $E_{a}=$
$=3.72 \pm 0.54 \mathrm{eV}, A=(3.84 \pm 0.60) \cdot 10^{15} \mathrm{~s}^{-1}$ для дефекта SWB. Воспользовавшись формулой (1), оценим времена отжига этих дефектов при температурах вне изученного диапазона. Для дефекта SWA и SWB найдем соответственно $\tau \sim 6 \mathrm{~ms}$ и $\sim 1 \mathrm{~s}$ с при $T=1200 \mathrm{~K}$, $\sim 0.1 \mathrm{~s}$ и $\sim 32 \mathrm{~s}$ при $T=1100 \mathrm{~K}, \sim 2 \mathrm{~s}$ и $\sim 27$ min при $T=1000$ К и т.д.

В отличие от фуллерена $\mathrm{C}_{60}$ мы ни разу не наблюдали образования новых дефектов SW при нагревании УНT. По-видимому, это связано с более высокими барьерами для их формирования. Между тем при $T>4000 \mathrm{~K}$ несколько раз имел место разрыв одной из связей C-C, вслед за которым трубчатая структура быстро разрушалась.
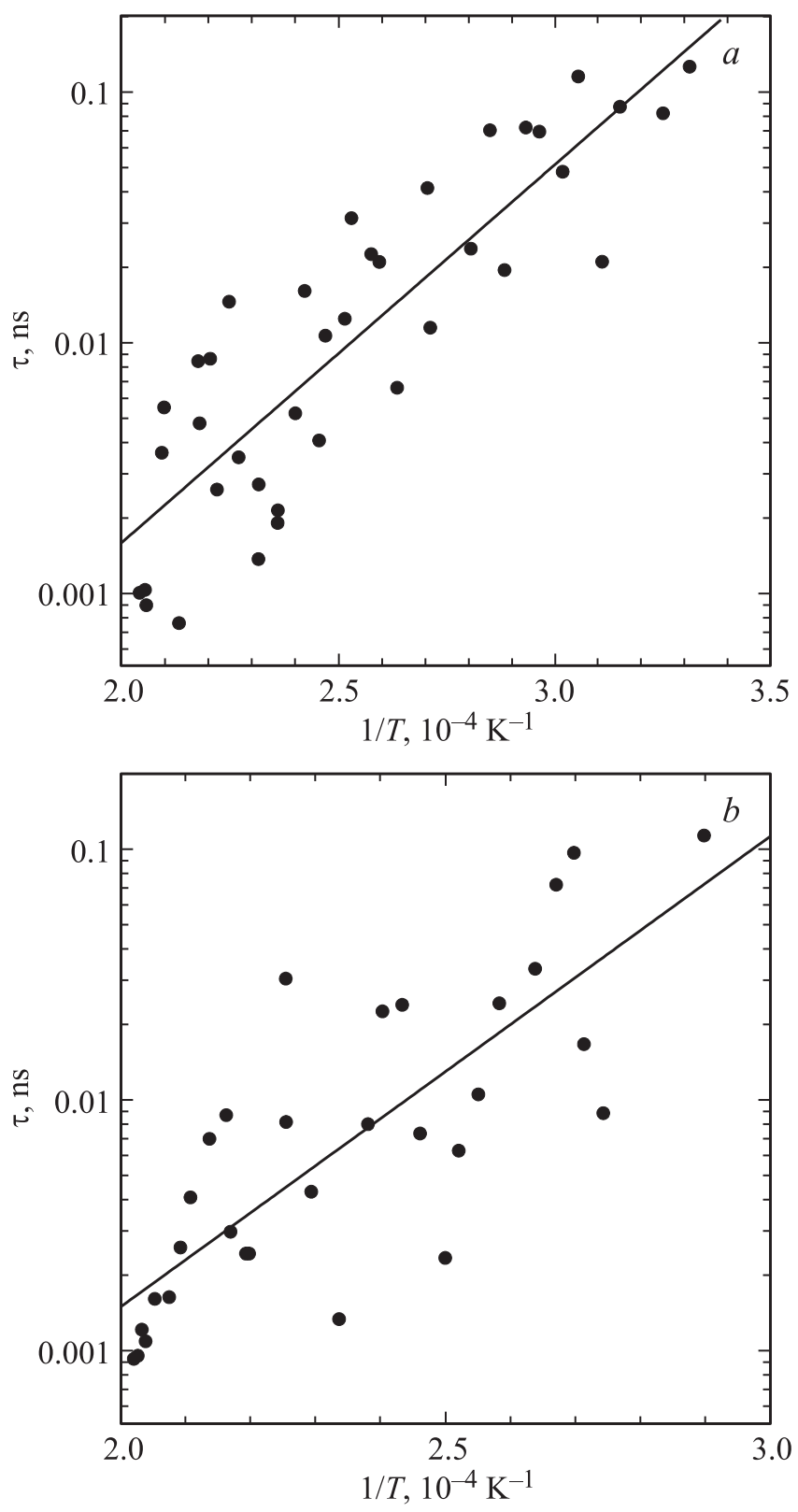

Pис. 6. То же, что на рис. 3 , для дефектов SWA $(a)$ и SWB $(b)$ в УНТ $(10,0)$, см. рис. 4. 


\section{4. Заключение и выводы}

На примере фуллерена $\mathrm{C}_{60}$ и нанотрубки $(10,0)$ показано, что нагревание углеродных наноструктур до высоких температур приводит к отжигу дефектов Стоуна-Уэльса. Время отжига сильно зависит от температуры, увеличиваясь при ее понижении. Температура должна подбираться так, чтобы величина тепловой энергии была недостаточно высока для образования новых дефектов. Таким образом, термический отжиг представляет собой эффективный способ очистки углеродных наноструктур от топологических дефектов.

Понизить энергетические барьеры для отжига дефектов и сократить тем самым время отжига можно путем адсорбции на наноструктуру атомарного водорода или гидроксильных групп [29,30]. Нужно, однако, иметь в виду, что барьеры для формирования новых дефектов при этом тоже станут меньше.

\section{Список литературы}

[1] H.W. Kroto, J.R. Heath, S.C. O’Brien, R.F. Curl, R.E. Smalley. Nature 318, 162 (1985).

[2] S. Iijima. Nature 354, 56 (1991).

[3] K.S. Novoselov, A.K. Geim, S.V. Morozov, D. Jiang, Y. Zhang, S.V. Dubonos, I.V. Grigorieva, A.A. Firsov. Science 306, 666 (2004).

[4] Л.А. Опенов, А.И. Подливаев. ФТТ 58, 1646 (2016).

[5] A.J. Stone, D.J. Wales. Chem. Phys. Lett. 128, 501 (1986).

[6] Л.А. Опенов, А.И. Подливаев. Письма в ЖЭТФ 84, 73 (2006).

[7] Q. Lu, B. Bhattacharya. Nanotechnology 16, 555 (2005).

[8] L. Pan, Z. Shen, Y. Jia, X. Dai. Physica B 407, 2763 (2012).

[9] D. Tasis, N. Tagmatarchis, A. Bianco, M. Prato. Chem. Rev. 106, 1105 (2006).

[10] C. Wang, G. Zhou, H. Liu, W. Duan. J. Phys. Chem. B 110, 10266 (2006).

[11] С.С. Моливер, Р.Р. Зимагуллов, А.Л. Семенов. Письма в ЖТФ 37, 68 (2011).

[12] M.M. Maslov, A.I. Podlivaev, K.P. Katin. Molecular Simulation 42, 305 (2016).

[13] K.P. Katin, V.S. Prudkovskiy, M.M. Maslov. Physica E 81, 1 (2016).

[14] Л.А. Опенов, А.И. Подливаев. Письма в ЖЭТФ 104, 192 (2016).

[15] Л.А. Опенов, А.И. Подливаев. ФТТ 58, 821 (2016).

[16] M.M. Maslov, K.P. Katin. Chem. Phys. Lett. 644, 280 (2016).

[17] Л.А. Опенов, А.И. Подливаев. Письма в ЖТФ 36, 69 (2010).

[18] J.-Y. Yi, J. Bernholc. J. Chem. Phys. 96, 8634 (1992).

[19] R.L. Murry, D.L. Strout, G.K. Odom, G.E. Scuseria. Nature 366, 665 (1993).

[20] R.L. Murry, D.L. Strout, G.E. Scuseria. Int. J. Mass Spectrom. Ion Proc. 138, 113 (1994).

[21] B.R. Eggen, M.I. Heggie, G. Jungnickel, C.D. Latham, R. Jones, P.R. Briddon. Science 272, 87 (1996).

[22] Y. Kumeda, D.J. Wales. Chem. Phys. Lett. 374, 125 (2003).

[23] H.F. Bettinger, B.I. Yakobsen, G.E. Scuseria. J. Am. Chem. Soc. 125, 5572 (2003).
[24] G.V. Vineyard. J. Phys. Chem. Solids. 3, 121 (1957).

[25] А.И. Подливаев, Л.А. Опенов. Письма в ЖЭТФ 81, 656 (2005).

[26] E. Ertekin, D.C. Chrzan, M.S. Daw. Phys. Rev. B 79, 155421 (2009).

[27] M. Kabir, K.J. Van Vliet. J. Phys. Chem. C 120, 1989 (2016).

[28] L.G. Zhou, S.-Q. Shi. Appl. Phys. Lett. 83, 1222 (2003).

[29] J.-Y. Yi, J. Bernholc. Chem. Phys. Lett. 403, 359 (2005).

[30] A.J.M. Nascimento, R.W. Nunes. Nanotechnology 24, 435707 (2013). 